\title{
Implant boosts activity in injured brain
}

Brain function has been improved in a patient who was in a minimally conscious state, by electrically stimulating a specific brain region with implanted electrodes. The achievement raises questions about the treatment of other patients who have been in this condition for years, the researchers say.

Patients in a minimally conscious state, often the result of severe brain trauma, show only intermittent evidence of awareness of the world around them. Typically, they are assumed to have little chance of further recovery if they show no improvement during their initial 12month rehabilitation programme.

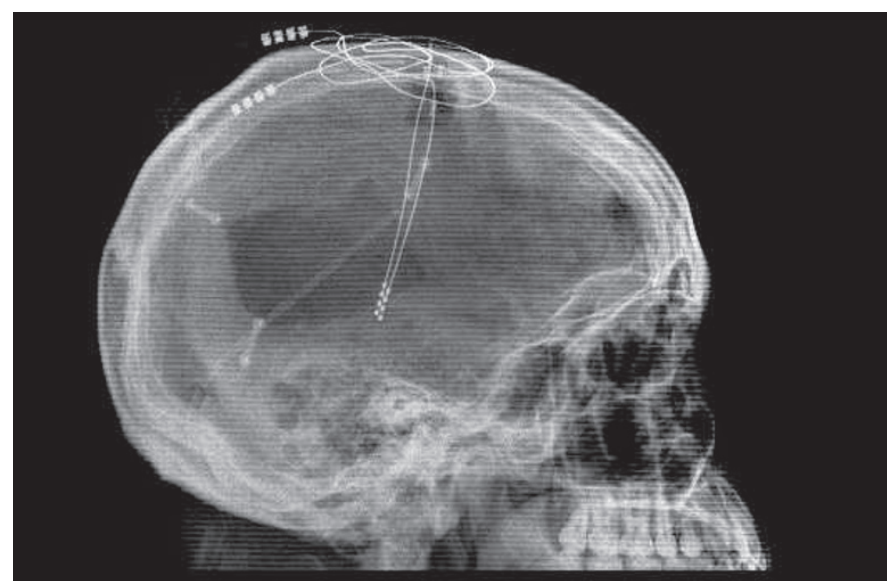

Deep-brain stimulation might help trauma patients regain consciousness.

who will show recovery.

Nevertheless, the case shows that many patients currently seen as beyond hope of rehabilitation might benefit from the results of further research. "Severe brain injury is not an uncommon problem, and the number of people doing research on this is shockingly small," Schiff says. "It's very rare to find a programme that will take a patient in a minimally conscious state even straight out of acute care. If they don't respond in a lively enough way and can't communicate and interact with people at the bedside, they go to a nursing home directly."

In the latest case study, neuroscientists describe how they implanted electrodes in the brain of a 38-year-old man who had been in a minimally conscious state for more than six years following a serious assault. By electrically stimulating a brain region called the central thalamus, they were able to help him name objects on request, make precise hand gestures, and chew food without the aid of a feeding tube (see page 600). The thalamus is involved in motor control, arousal and in relaying sensory signals - from the visual systems, for example - to the cerebral cortex, the part of the brain involved in consciousness.

"There will be a subset of patients who are responsive to this approach."
Nicholas Schiff of Weill Cornell Medical College in New York, and his colleagues chose the patient because they believed his condititon was due to impairment of the arousal system, and that despite considerable damage to his cerebral cortex, many essential areas were preserved.

"There will be a subset of patients who are responsive to this approach," says Schiff. But he adds that patients with different brain injuries may not benefit from electrostimulation. "Not every patient in a minimally conscious state will fit this profile," Schiff says, and it is difficult for neurologists to identify those patients
"The report does not suggest that deepbrain stimulation [DBS] 'cures' the minimally conscious state," says Paul Matthews, a clinical neuroscientist at Imperial College, London. "Although based on a study of only a single patient, it suggests that DBS may be adapted to benefit at least some patients in the minimally conscious state. And it emphasizes that improvements can be made by patients even long after an injury.

"Although we do not know precisely which brain connections are important, we may expect that some specific connections must be intact for DBS to have a beneficial effect." Michael Hopkin

See also News \& Views, page 539.

\section{Flatworms' starring role in stem-cell research}

Tiny channels provide corridors of communication between adult stem cells and their differentiated neighbours, a study has found. The finding could be an important step towards determining how to control the development of adult stem-cell identity, but there's a catch - it was discovered in the planarian flatworm Schmidtea mediterranea.

The flatworms, best known for their regenerative prowess and cross-eyed charm, maintain a supply of stem cells to regrow tissue lost to anything from daily wear-and-tear to full decapitation. And, although it seems a long way to travel along the evolutionary tree, there are reasons to think planarians make a good model for human stem cells, says regeneration biologist and planarian researcher Alejandro Sánchez Alvarado at the University of Utah School of Medicine in Salt Lake City. He belives that the basic principles uncovered in planarians will translate to human cells, although the finer details may differ.

Even biologists who don't study the worms agree. "Work in planarians and other lower organisms always gives us hints regarding human stem cells," says Shinya Yamanaka, a stem-cell researcher at Kyoto University in Japan.
Biologists Néstor Oviedo and Michael Levin of the Forsyth Institute in Boston, Massachusetts, studied channels known as gap junctions that permit the passage of small molecules and ions between cells. When S. mediterranea was manipulated so that it did not express a key gap-junction protein, thus preventing communication between cells, it lost its regenerative capabilities and its reservoir of stem cells (N. J. Oviedo and M. Levin Development 134, 3121-3131; 2007).

This fits with the findings of studies in human embryonic stem cells, says Alice Pébay, a biologist at the University of Melbourne,
Australia. Previous work has shown that chemically induced closure of gap junctions can slow human embryonic stem-cell growth. But, notes Pébay, Ovideo and Levin were able to use a technique to reduce expression of the gap-junction proteins - something that would be very difficult to do in human embryonic stem cells.

Nevertheless, technical challenges to planarian research remain. For example, reducing expression of an S. mediterranea gene is straightforward, but techniques for over-expressing planarian genes are still rudimentary, says Levin. Heidi Ledford 